Review

\title{
DJ-1 in Ocular Diseases: A Review
}

\author{
Cong Liu*, Xiufen Liu*, Jing Qi, Om Prakash Pant, Cheng-wei Lu ${ }^{\bowtie}$ and Jilong Hao ${ }^{\bowtie}$ \\ Department of Ophthalmology, The First Hospital of Jilin University, Jilin, China. \\ *Cong Liu and Xiufen Liu contribute equally to this manuscript. \\ $\bowtie$ Corresponding authors: Cheng-wei Lu, lcwchina800@sina.com and Jilong Hao, 289736582@qq.com \\ (c) Ivyspring International Publisher. This is an open access article distributed under the terms of the Creative Commons Attribution (CC BY-NC) license \\ (https://creativecommons.org/licenses/by-nc/4.0/). See http://ivyspring.com/terms for full terms and conditions.
}

Received: 2017.10.21; Accepted: 2018.01.05; Published: 2018.02.12

\begin{abstract}
Protein deglycase DJ-1 (Parkinson disease protein 7) is a $20 \mathrm{kDa}$ protein encoded by PARK7 gene. It is also known as a redox-sensitive chaperone and sensor that protect cells against oxidative stress-induced cell death in many human diseases. Though increasing evidence implicates that DJ-1 may also participate in ocular diseases, the overview of DJ-1 in ocular diseases remains elusive. In this review, we discuss the role as well as the underlying molecular mechanisms of DJ-1 in ocular diseases, including Fuchs endothelial corneal dystrophy (FECD), age-related macular degeneration (AMD), cataracts, and ocular neurodegenerative diseases, highlighting that $\mathrm{DJ}-1$ may serve as a very striking therapeutic target for ocular diseases.
\end{abstract}

Key words: DJ-1, ocular disease, oxidative stress

\section{Introduction}

Protein deglycase DJ-1 is coded by the human PARK7 gene on chromosome 1 (1p36.12-1p36.33) [1], and also named as Parkinson disease protein 7 due to the fact that DJ-1 was first discovered in Parkinson's disease (PD) patients [2]. DJ-1 is a member of the DJ-1/Hsp31/PfpI superfamily [3]. With a size of 20 kDa [4], DJ-1 consists of 189 amino acids ( 9 a-helices and $7 \beta$-strands in total) [5] and it is usually presented as a dimer which is important for its biological activity. Mutations destroy the dimer structure of DJ-1. For instance, L166P and M26I weaken its biological activity [6].

The DJ-1 protein is abundantly expressed in more than 22 human tissues [7]. DJ-1 is associated with multiple biological functions, such as mitochondrial function regulation [8], transcriptional regulation, molecular chaperone [9], glyoxalase [10], cysteine protease regulation, glutathione regulation $[11,12]$, dopamine regulation [13], and the subunit of RNA-binding protein regulation. Of most importance, dependable findings revealed that DJ-1 possesses an antioxidant activity and plays a role as a redox activated chaperone [9] in cytoprotective function under stimuli challenge [14, 15]. DJ-1 has 3 cysteine residues, Cys-106, Cys-46 and Cys-53, with Cys-106 being the active site [16]. DJ-1 may exhibit its anti-oxidative defence through oxidation of Cys-106 to regulate transcription factors instead of removing ROS directly.

DJ-1, an important endogenous antioxidant, is expected to be a target of mechanism-oriented therapy for neurodegenerative diseases, cardiovascular diseases, and cancers [17]. Many ocular diseases are associated with oxidative stress but DJ-1 is never reviewed in ocular diseases. In this review, we discuss the role of DJ-1 in the pathogenesis of ocular diseases and the mechanism to target DJ-1 for treatment of these diseases.

\section{Fuchs endothelial corneal dystrophy (FECD)}

FECD is a severe corneal dystrophy with slow, progressive loss of corneal endothelial cells resulting in corneal edema and vision loss at the late stage. Currently, corneal transplantation is the mainstream treatment option to restore the vision [18]. Oxidative stress has been considered to exert an important role in the apoptosis of corneal endothelial cells (CECs) in FECD. Nuclear factor erythroid 2-related factor 2 
(Nrf2) is a foremost transcription regulator of cellular protector against oxidative stress. DJ-1 is a protein stabilizer of Nrf2 and was markedly decreased at both mRNA and protein levels of CECs in FECD. Under oxidative stress, DJ-1 protein synthesis was significantly up-regulated in normal CECs. Whereas decreased levels of DJ-1 was found in FECD at baseline, which-diminished Nrf2 nuclear transloncation and accelerated CECs apoptosis. The pathway of DJ-1/Nrf2 axis could serve as a new therapeutic target of FECD by slowing CEC degeneration in FECD [19]. Sulforaphane (SFN) is thought as an "indirect antioxidant" and can augment effects on varieties of antioxidant genes. In Alireza Ziaei et Al. study, SFN enhanced nuclear translocation of Nrf2 in human FECD specimens under tert-Butyl hydroperoxide (tBHP) induced oxidative stress and led to increased DJ-1 synthesis. This proof-of-concept investigation indicated that DJ-1 may protect FECD against oxidative stress by activating Nrf2-antioxidant response element (ARE) pathway [20]. Ultraviolet A (UV-A), with a wavelength of 320-400 nm can initiate cell apoptosis indirectly by producing reactive oxygen species (ROS). Small interfering RNA (siRNA) of DJ-1 was transfected into the normal human corneal endothelial cell line (HCECi) to obtain the DJ-1-deficient CECs. Increased ROS overproduction and decreased DJ-1, nuclear Nrf2 proteins were found in DJ-1 siRNA-treated cells when compared to controls. DJ-1-deficient CECs exhibited a vulnerable response to UV-A irradiation, and the declined in DJ-1 led to activation of caspase- 3 and phospho-p53 under the oxidative stress. And plays a central role in the execution-phase of cell apoptosis, indicating that DJ-1 may serve a protective role against UV-A-induced apoptosis by inhibiting phospho-53-mediated apoptosis pathway. Down-regulation of DJ-1 also attenuated Nrf2 nuclear translocation, causing the decreasement of transcription of antioxidant genes (NQO1 and HO-1) in DJ-1-deficient CECs and weakened antioxidant defense [19].

DJ-1 may serve as a potential treatment option for corneal endothelial disorders, such as FECD. Further studies are required to understand the role of DJ-1 in FECD more precisely.

\section{Age-related macular degeneration (AMD)}

AMD is a common retinal disease and also a leading cause of irreversible vision loss in the old patients. The degeneration of retinal pigment epithelium (RPE) cells, which is the pigmented cell layer just outside the neurosensory retina that nourishes retinal visual cells, in association with oxidative stress is related to AMD pathogenesis.

The photoreceptors and RPE in DJ-1-deficient retinas exhibited signs of physiological dysfunction, accompanied by the increased carbonyl content (protein oxidation product), nitric oxide synthase (iNOS, an oxidative stress marker) and decreased Nrf2 expression (antioxidant transcriptional regulator) in retina/RPE lysates of DJ-1 $\mathrm{KO}$ mice. Moreover, a single tail vein injection of $\mathrm{NaIO} 3$ (an oxidizing reagent) significantly accelerated RPE degeneration in DJ-1 KO mice [21]. Reduced photoreceptor activity was also found by the ERG in old DJ-1 KO retinas when compared to age-matched controls. These results suggest that DJ-1 is a protecting factor for RPE/photoreceptors against the oxidative stress (such as ageing) by regulating Nrf2 signaling. Therefore, DJ-1 may serve as a potential target for the prevention of the geographic atrophy and vision loss in AMD [22].

\section{Cataract}

Cataract is the leading cause of blindness worldwide. Increasing age is considered as the main contributor to cataract, and the ageing eyes are susceptible to be at extensive risk to oxidative stress [23]. DJ-1 with oxidized cysteine at vital sites was also found to be dramatically expressed in lens fibers [24].

The experiment demonstrated that the cysteine residues of DJ-1, Cys46 and Cys53, were found to be oxidized in aged cataractous human lenses. Similar results were also found in glutathione-depleted mouse (LEGSKO) lenses and in vitro oxidation model triggered by $\mathrm{H}_{2} \mathrm{O}_{2}$ when compared to controls. As long-lived proteins, the lens proteins are inclined to damage accumulation. Substantial evidence points out that cataract is associated with disulfide-linked high molecular weight crystal aggregation. The oxidation of DJ-1 protein was closely related to disulfide cross-linking. The disulfide ratio of the Cys46 and Cys53 in an aged human lens is much higher than the controls. Similar results were also detected in Cys46 and Cys53 oxidation in LEGSKO vs. age-matched mouse lens and in vitro modelling samples.

The above results indicate that DJ-1 is a significant oxidation site in the lens via the disulfide cross-linking [25].

\section{Ocular neurodegenerative diseases}

Ocular neurodegenerative diseases, such as glaucoma, optic neuropathies are characterized by the damage of the optic nerve as well as progressive deterioration of retinal ganglion cells (RGCs), which constitute significant elements for chronic visual injury [26-28]. Oxidative stress plays an important role in the pathogenesis of ocular neurodegenerative diseases [29]. Glutathione level, a significant 
antioxidant in the retina, is decreased markedly in plasma of glaucoma patients [30,31]. The antioxidant drug, Geranylgeranylacetone (GGA) is effective in protecting RGCs in glaucoma mouse models [32]. Optic neuritis (ON), an optic nerve disease, is strongly associated with inflammatory demyelination [33] and present symptoms in $20 \%$ of multiple sclerosis (MS) patients [34]. Oxidative stress is indicated to play a key role in the pathogenesis of MS [35] and antioxidants are demonstrated to be effective in ameliorating the inflammation of the optic nerve. For instance, natural antioxidant lipoic acid [36] and spermidine [37] are effective in suppressing inflammation and protecting RGCs in the optic nerve of experimental autoimmune encephalomyelitis (EAE) mice. These findings suggest that antioxidants may serve as effective treatment options for ocular neurodegenerative diseases. DJ-1 regulates varieties of signal transductions related to oxidative stress and may exhibit anti-oxidative effects as well as protect RGCs via various signaling pathways such as Nrf2 pathway, PI3K/Akt pathway and ASK1 pathway.

\section{Nuclear factor erythroid 2-related factor $\mathbf{2}$ (Nrf2) pathway}

Nrf2 is a master transcription factor associated with oxidative stress and can modify the basal and inducible expressions of several antioxidant genes [38]. Nrf2 KO mice are vulnerable to the ocular diseases and are related to oxidative stress. Genetic ablation of Nrf2 can aggravate irreversible RGCs apoptosis and visual deficits in the murine model [39].Gene therapy with Nrf2 could reduce RGCs degeneration [40]. DJ-1 stabilizes Nrf2 and induces the expression of antioxidant thioredoxin 1 through the Nrf2 pathway [41]. These findings above indicate that DJ-1 could be a potential therapeutic target for ocular neurodegenerative diseases via regulating the activation of Nrf2.

\section{PI3K/Akt pathway}

The phosphoinositide 3-kinase (PI3K)/Akt signaling pathway plays an important part in blocking oxidative stress [42] and functions as a neuroprotective effect for the injured RGCs [43]. Activation of the PI3K/Akt pathway provides a possible therapeutic target for RGCs damage in retinal ischemia [44, 45]._Phosphatase and Tensin homolog deleted on chromosome 10 (PTEN) is a negative regulator of PI3K/Akt pathway [46]. DJ-1 activated PI3K/Akt pathway and exhibited its cytoprotective role via the inhibition of PTEN $[47,48]$ indicating that DJ-1 may serve as a strategy to achieve neuroprotection of RGCs in ocular neurodegenerative diseases.

\section{ASK1 pathway}

Apoptosis signal-regulating kinase 1 (ASK1) is a member of the stress-responsive mitogen-activated protein kinase kinase kinase (MAP3K) family and plays a vital role in regulating oxidative stress [49] or cytokine-induced apoptosis [50]. ASK1 is activated by the phosphorylation of a vital threonine residue in the oxidative stress state [51]. ASK1 deletion reduced the oxidative stress level and the factors which cause oxidative stress, for instance, TNF-a [52]. ASK1 deletion prevented RGC apoptosis and increased RGC survival in mice model of glaucoma [53]. This experiment shows that ASK1 is a negative factor for RGC, and ASK1 inhibition can be an effective target for treatment of glaucoma. Under oxidative stress, DJ-1 binds with ASK1 via the Cys-106 and forms the mixed disulfide bonds, which provide cytoprotection in mouse embryonic fibroblast [54, 55]. These results indicate that DJ-1 may provide a promising approach for the treatment of glaucoma.

The above pathway describes the role of DJ-1 in the protection of RGCs. DJ-1- related treatment, by targeting oxidative stress could be a promising step in the management of ocular neurodegenerative diseases.

\section{Uveal Melanoma (UM)}

DJ-1 was found as a putative oncogenic gene years ago [56]. Accumulate evidence have shown that DJ-1 is frequently overexpressed and secreted in several tumor cells, for example, prostate cancer [57], hepatocellular carcinoma [58], non-small cell lung carcinoma (NSCLC) [59], laryngeal squamous cell carcinoma [60], and esophageal squamous cell carcinoma (ESCC) [61], indicating that DJ-1 overexpression is a regular event in cancer cells.

Choroidal nevi, which is referred to as pigmented lesions are usually benign. However, Choroidal nevi may develop to UM, and the risk of malignant transformation is estimated $0.011 \%$.

Elevated serum levels of DJ-1 in choroidal nevi patients had a significant correlation with clinical risk factors of malignant transformation (e.g. nevus thickness $>1.5 \mathrm{~mm}$, a large basal diameter $>8 \mathrm{~mm}$ ). DJ-1 level was shown to have a positive correlation with clinical risk factors for choroidal nevi growth and may be a promising biomarker of malignancy [62].

$\mathrm{UM}$, is a cancer of the melanocytes in the uvea (the iris, ciliary body, and choroid) of the eye. It is considered as the most frequent primary intraocular malignant growth in adults with high risk of blood disseminating and hepatic metastases [63, 64]. UM-A, a primary and well-characterized UM cell, is proved 
to be a valuable cell model for studying UM in vitro. DJ-1 protein could be secreted by both UM-A and other UM cell lines cultured in vitro, but not by normal melanocytes, indicating that DJ-1 protein may serve as a potential serum marker for UM [65]. DJ-1 level in serum of patients with metastatic UM was reported to be significantly upregulated, either compared with UM disease-free controls (at last 10 years following primary UM treatment) or compared with normal healthy controls. The study further indicated that overexpression of DJ-1 may be associated with metastatic UM. Consequently, DJ-1 could be a potential serological biomarker for detection of UM metastases in patients at an early stage $[66,67]$.

To summarize, DJ-1 is closely related to the occurrence and development of UM and can be used as a biomarker for UM diagnosis and prognosis evaluation. However, the mechanism is still not clear and more investigations are still needed.

\section{Conclusions}

DJ-1 is expressed in various ophthalmological diseases and the function of DJ-1, as well as the underlying pathway involved varies in different diseases. As discussed above, DJ-1 plays a role as an antioxidant in FECD, AMD, cataracts, and ocular neurodegenerative diseases. Both in vitro and in vivo studies showed that it may exert protective role through various pathways (Nrf2 pathway, PI3K/Akt pathway, ASK1 pathway). Furthermore, DJ-1 is overexpressed in the progression of UM and can be used as a biomarker for UM diagnosis. Thus, DJ-1 could be a promising target directing the future treatment of related ocular diseases.

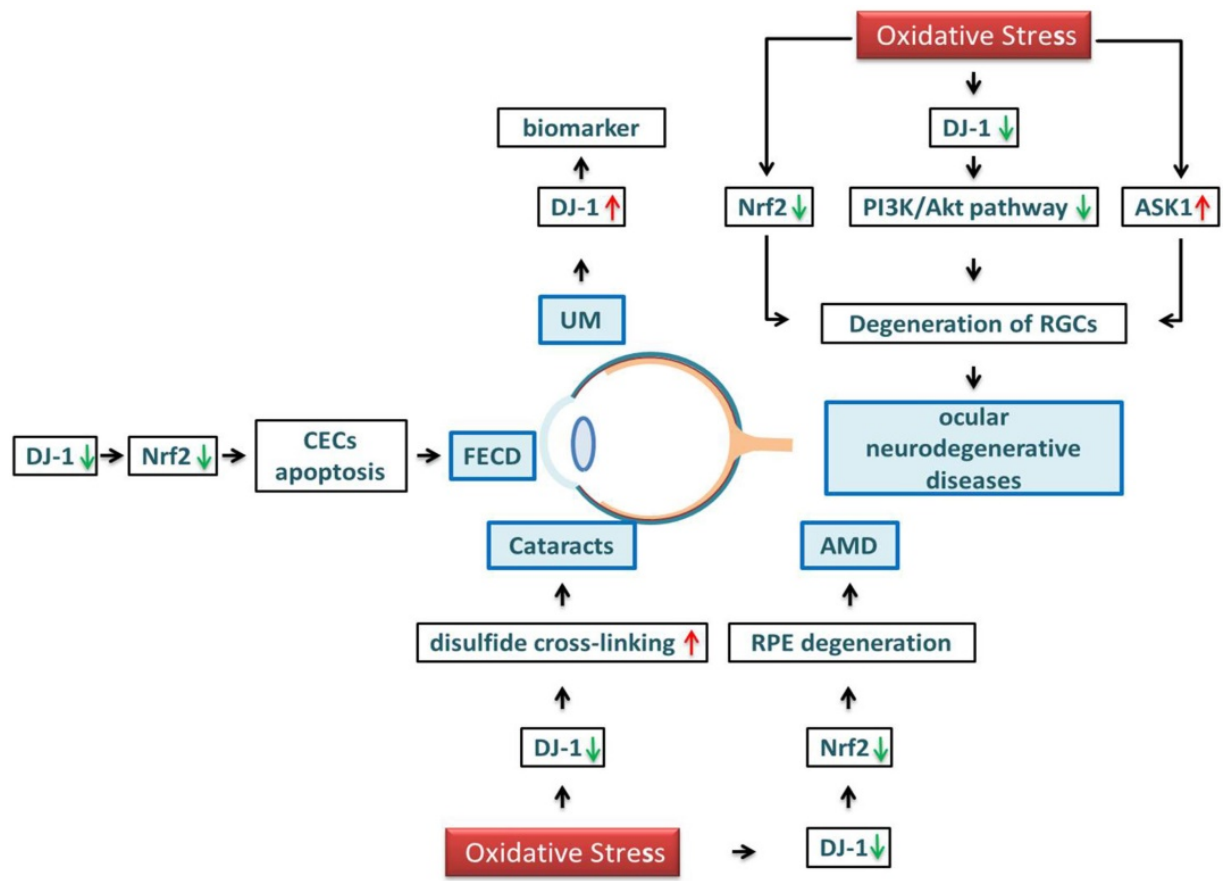

Figure 1. Schematic diagram of DJ-1 regulation in ocular diseases. Under oxidative stress, the level of DJ-1 and Nrf2 are decreased in the cornea, causing the CECs apoptosis and leading to the reduction of FECD. DJ-1 and Nrf2 in the lens, resulting in the formation of a disulfide bond, crystal turbidity, and cataract formation. DJ-1 and Nrf2 are reduced in the retina, accelerating RPE degeneration and leading to AMD. Oxidative stress also induces the downregulation of Nrf2, DJ-1/PI3K/Akt and activation of ASK1, leading to the aggravation of irreversible RGCs apoptosis, resulting in ocular neurogeneration diseases. The expression level of DJ-1 in UM is significantly upregulated, DJ-1 protein may serve as a potential serum marker for UM.

Table 1. Selected studies on the relationship between DJ-1 inducer/suppressor and ocular diseases

\begin{tabular}{|c|c|c|c|c|}
\hline Ocular Disease & Type & Models & Results & Reference \\
\hline \multirow[t]{2}{*}{ FECD } & In vitro & $\begin{array}{l}\text { Human corneal } \\
\text { endothelial cell lines }\end{array}$ & $\begin{array}{l}\text { SFN enhanced nuclear translocation of Nrf2 in human FECD specimens under tBHP induced } \\
\text { oxidative stress and led to increased DJ-1 synthesis. }\end{array}$ & [20] \\
\hline & In vitro & $\begin{array}{l}\text { CECs from ex vivo } \\
\text { corneas of DJ-1 } \\
\text { knockout mice }\end{array}$ & $\begin{array}{l}\text { DJ-1-deficient CECs exhibited the vulnerable response to UV-A irradiation, and the decline in } \\
\text { DJ-1 led to activation of caspase- } 3 \text { and phospho-p } 53 \text { under the oxidative stress, which plays a } \\
\text { central role in the execution-phase of cell apoptosis. } \\
\text { DJ-1 may serve a protective role against UV-A-induced apoptosis by inhibiting } \\
\text { phospho-53-mediated apoptosis pathway. }\end{array}$ & [19] \\
\hline AMD & In vivo & DJ-1 KO mice & $\begin{array}{l}\text { DJ-1 is a protecting factor for RPE/photoreceptors against the oxidative stress (such as aging) } \\
\text { by regulating Nrf2 signaling and DJ-1 may serve as a potential target for the prevention of the } \\
\text { geographic atrophy and vision loss in AMD. }\end{array}$ & {$[21,22]$} \\
\hline
\end{tabular}




\begin{tabular}{llll}
\hline Ocular Disease & Type & Models & Results \\
\hline Cataracts & In vivo & $\begin{array}{l}\text { cataractous human } \\
\text { lens; } \\
\text { glutathione-depleted }\end{array}$ & $\begin{array}{l}\text { Cys46 and Cys53 were found to be oxidized in aged cataractous human lens and } \\
\text { glutathione-depleted mouse. } \\
\text { The oxidation of DJ-1 protein was closely related to disulfide cross-linking. }\end{array}$ \\
& & [25].
\end{tabular}

ocular In vivo Nrf2 $\mathrm{KO}$ mice

neurodegenerative

diseases

RGC apoptosis is considerably increased in Nrf2 KO mice, and gene therapy with Nrf2 can reduce RGC death.

DJ-1 can stabilize Nrf2 and induce the expression of antioxidant thioredoxin 1 through the Nrf2 pathway.

In vitro Mouse NIH3T3 cells Activation of the PI3K/Akt pathway is often induced by substances able to prevent RGC death [47, 48] in retinal ischemia.

DJ-1 activated PI3K/Akt pathway and exhibited its cytoprotective role via the inhibition of PTEN.

In vivo mice model of ASK1 deletion prevented RGC apoptosis and increased RGC survival in mice model of

glaucoma

In vitro mouse embryonic DJ-1 can bind with ASK1 via the Cys-106 under oxidative stress.

UM In vitro UM cell lines

DJ-1 protein could be secreted into the bloodstream by both UM-A and other UM cell lines cultured in vitro, but not by normal melanocytes, indicating that DJ-1 protein may serve as a potential serum marker for UM.

Abbreviations: FECD, Fuchs endothelial corneal dystrophy; SFN, Sulforaphane; AMD, Age-related macular degeneration; Nrf2, Nuclear factor erythroid 2-related factor 2; tBHP, tert-Butyl hydroperoxide; CECs, corneal endothelial cells; PI3K, phosphoinositide 3-kinase; RGC, retinal ganglion cells; PTEN, Phosphatase and Tensin homolog deleted on chromosome 10; ASK1, Apoptosis signal-regulating kinase 1; UM, uveal melanoma

\section{Competing Interests}

The authors have declared that no competing interest exists.

\section{References}

1. Taira T, Takahashi K, Kitagawa R, et al. Molecular cloning of human and mouse DJ-1 genes and identification of Sp1-dependent activation of the human DJ-1 promoter. Gene. 2001;263(1-2): 285-292.

2. Bonifati V, Rizzu P, van Baren MJ, et al. Mutations in the DJ-1 gene associated with autosomal recessive early-onset parkinsonism. Science. 2003;299(5604): 256-259.

3. Tao X, Tong L. Crystal structure of human DJ-1, a protein associated with early onset Parkinson's disease. J Biol Chem. 2003;278(33): 31372-31379.

4. Honbou K, Suzuki NN, Horiuchi M, et al. The crystal structure of DJ-1, a protein related to male fertility and Parkinson's disease. J Biol Chem. 2003;278(33): 31380-31384.

5. Huai Q, Sun Y, Wang H, et al. Crystal structure of DJ-1/RS and implication on familial Parkinson's disease. FEBS Lett. 2003;549(1-3): 171-175.

6. Lee SJ, Kim SJ, Kim IK, et al. Crystal structures of human DJ-1 and Escherichia coli Hsp31, which share an evolutionarily conserved domain. J Biol Chem. 2003;278(45): 44552-44559.

7. Hod Y, Pentyala SN, Whyard TC, et al. Identification and characterization of a novel protein that regulates RNA-protein interaction. J Cell Biochem. 1999;72(3): 435-444.

8. Wang X, Petrie TG, Liu Y, et al. Parkinson's disease-associated DJ-1 mutations impair mitochondrial dynamics and cause mitochondrial dysfunction. J Neurochem. 2012;121(5): 830-839.

9. Zhou W, Zhu M, Wilson MA, et al. The oxidation state of DJ-1 regulates its chaperone activity toward alpha-synuclein. J Mol Biol. 2006;356(4): 1036-1048.

10. Wei $Y$, Ringe D, Wilson MA, et al. Identification of functional subclasses in the DJ-1 superfamily proteins. PLoS Comput Biol. 2007;3(1): e10.

11. Guzman JN, Sanchez-Padilla J, Wokosin D, et al. Oxidant stress evoked by pacemaking in dopaminergic neurons is attenuated by DJ-1. Nature. 2010;468(7324): 696-700.

12. Zhou W, Freed CR. DJ-1 up-regulates glutathione synthesis during oxidative stress and inhibits A53T alpha-synuclein toxicity. J Biol Chem. 2005;280(52): 43150-43158.

13. Zhong N, Kim CY, Rizzu P, et al. DJ-1 transcriptionally up-regulates the human tyrosine hydroxylase by inhibiting the sumoylation of pyrimidine tract-binding protein-associated splicing factor. J Biol Chem. 2006;281(30): 20940-20948.

14. Lev N, Ickowicz D, Barhum Y, et al. DJ-1 protects against dopamine toxicity. J Neural Transm (Vienna). 2009:116(2): 151-160.

15. Taira T, Saito $\mathrm{Y}, \mathrm{Niki} \mathrm{T}$, et al. DJ-1 has a role in antioxidative stress to prevent cell death. EMBO Rep. 2004;5(2): 213-218.

16. Blackinton $\mathrm{J}$, Lakshminarasimhan $\mathrm{M}$, Thomas KI, et al. Formation of a stabilized cysteine sulfinic acid is critical for the mitochondrial function of the parkinsonism protein DJ-1. J Biol Chem. 2009;284(10): 6476-6485.

17. Chan JY, Chan SH. Activation of endogenous antioxidants as a common therapeutic strategy against cancer, neurodegeneration and cardiovascular diseases: A lesson learnt from DJ-1. Pharmacol Ther. 2015;156: 69-74.
18. Vedana G, Villarreal G, Jr., Jun AS. Fuchs endothelial corneal dystrophy: current perspectives. Clin Ophthalmol. 2016;10: 321-330.

19. Liu C, Chen Y, Kochevar IE, et al. Decreased DJ-1 leads to impaired Nrf2-regulated antioxidant defense and increased UV-A-induced apoptosis in corneal endothelial cells. Invest Ophthalmol Vis Sci. 2014;55(9): 5551-5560.

20. Ziaei A, Schmedt T, Chen $Y$, et al. Sulforaphane decreases endothelial cell apoptosis in fuchs endothelial corneal dystrophy: a novel treatment. Invest Ophthalmol Vis Sci. 2013;54(10): 6724-6734.

21. Bonilha VL, Bell BA, Rayborn ME, et al. Loss of DJ-1 elicits retinal abnormalities, visual dysfunction, and increased oxidative stress in mice. Exp Eye Res. 2015;139: 22-36.

22. Bonilha VL, Bell BA, Rayborn ME, et al. Absence of DJ-1 causes age-related retinal abnormalities in association with increased oxidative stress. Free Radic Biol Med. 2017;104: 226-237.

23. Babizhayev MA, Yegorov YE. Reactive Oxygen Species and the Aging Eye: Specific Role of Metabolically Active Mitochondria in Maintaining Lens Function and in the Initiation of the Oxidation-Induced Maturity Onset Cataract--A Novel Platform of Mitochondria-Targeted Antioxidants With Broad Therapeutic Potential for Redox Regulation and Detoxification of Oxidants in Eye Diseases. Am J Ther. 2016;23(1): e98-117.

24. Sun J, Rockowitz S, Chauss D, et al. Chromatin features, RNA polymerase II and the comparative expression of lens genes encoding crystallins, transcription factors, and autophagy mediators. Mol Vis. 2015;21: 955-973.

25. Wang B, Hom G, Zhou S, et al. The oxidized thiol proteome in aging and cataractous mouse and human lens revealed by ICAT labeling. Aging Cell. 2017;16(2): 244-261.

26. Pascolini D, Mariotti SP. Global estimates of visual impairment: 2010. Br J Ophthalmol. 2012;96(5): 614-618.

27. Seki M, Lipton SA. Targeting excitotoxic/free radical signaling pathways for therapeutic intervention in glaucoma. Prog Brain Res. 2008;173: 495-510.

28. Osborne NN, del Olmo-Aguado S. Maintenance of retinal ganglion cell mitochondrial functions as a neuroprotective strategy in glaucoma. Curr Opin Pharmacol. 2013;13(1): 16-22.

29. Goyal A, Srivastava A, Sihota R, et al. Evaluation of oxidative stress markers in aqueous humor of primary open angle glaucoma and primary angle closure glaucoma patients. Curr Eye Res. 2014;39(8): 823-829.

30. Gherghel D, Griffiths HR, Hilton EJ, et al. Systemic reduction in glutathione levels occurs in patients with primary open-angle glaucoma. Invest Ophthalmol Vis Sci. 2005;46(3): 877-883.

31. Gherghel D, Mroczkowska S, Qin L. Reduction in blood glutathione levels occurs similarly in patients with primary-open angle or normal tension glaucoma. Invest Ophthalmol Vis Sci. 2013;54(5): 3333-3339.

32. Dong Z, Shinmei Y, Dong Y, et al. Effect of geranylgeranylacetone on the protection of retinal ganglion cells in a mouse model of normal tension glaucoma. Heliyon. 2016;2(10): e00191.

33. Kimura A, Namekata K, Guo X, et al. Targeting Oxidative Stress for Treatment of Glaucoma and Optic Neuritis. Oxid Med Cell Longev. 2017;2017: 2817252.

34. Andersen MR, Roar M, Sejbaek T, et al. Long-term structural retinal changes in patients with optic neuritis related to multiple sclerosis. Clin Ophthalmol. 2017:11: 1519-1525.

35. Gilgun-Sherki Y, Melamed E, Offen D. The role of oxidative stress in the pathogenesis of multiple sclerosis: the need for effective antioxidant therapy. J Neurol. 2004;251(3): 261-268.

36. Chaudhary P, Marracci G, Yu X, et al. Lipoic acid decreases inflammation and confers neuroprotection in experimental autoimmune optic neuritis. J Neuroimmunol. 2011;233(1-2): 90-96. 
37. Guo X, Harada C, Namekata K, et al. Spermidine alleviates severity of murine experimental autoimmune encephalomyelitis. Invest Ophthalmol Vis Sci. 2011;52(5): 2696-2703.

38. Jung KA, Kwak MK. The Nrf2 system as a potential target for the development of indirect antioxidants. Molecules. 2010;15(10): 7266-7291.

39. Larabee CM, Desai S, Agasing A, et al. Loss of Nrf2 exacerbates the visual deficits and optic neuritis elicited by experimental autoimmune encephalomyelitis. Mol Vis. 2016;22: 1503-1513.

40. Liao G, Li R, Chen X, et al. Sodium valproate prevents radiation-induced injury in hippocampal neurons via activation of the Nrf2/HO-1 pathway. Neuroscience. 2016;331: 40-51.

41. Im JY, Lee KW, Woo JM, et al. DJ-1 induces thioredoxin 1 expression through the Nrf2 pathway. Hum Mol Genet. 2012;21(13): 3013-3024.

42. Manning BD, Cantley LC. AKT/PKB signaling: navigating downstream. Cell. 2007;129(7): 1261-1274

43. Nakazawa T, Shimura M, Tomita $H$, et al. Intrinsic activation of PI3K/Akt signaling pathway and its neuroprotective effect against retinal injury. Curr Eye Res. 2003;26(1): 55-63.

44. Qi Y, Chen L, Zhang L, et al. Crocin prevents retinal ischaemia/reperfusion injury-induced apoptosis in retinal ganglion cells through the PI3K/AKT signalling pathway. Exp Eye Res. 2013;107: 44-51.

45. Yang X, Huo F, Liu B, et al. Crocin Inhibits Oxidative Stress and Pro-inflammatory Response of Microglial Cells Associated with Diabetic Retinopathy Through the Activation of PI3K/Akt Signaling Pathway. J Mol Neurosci. 2017;61(4): 581-589.

46. Salmena L, Carracedo A, Pandolfi PP. Tenets of PTEN tumor suppression. Cell. 2008;133(3): 403-414.

47. Kim RH, Peters M, Jang $\mathrm{Y}$, et al. DJ-1, a novel regulator of the tumor suppressor PTEN. Cancer Cell. 2005;7(3): 263-273.

48. Kim YC, Kitaura H, Taira T, et al. Oxidation of DJ-1-dependent cell transformation through direct binding of DJ-1 to PTEN. Int J Oncol. 2009;35(6): 1331-1341.

49. Hattori K, Naguro I, Runchel C, et al. The roles of ASK family proteins in stress responses and diseases. Cell Commun Signal. 2009;7: 9.

50. Kawarazaki Y, Ichijo H, Naguro I. Apoptosis signal-regulating kinase 1 as a therapeutic target. Expert Opin Ther Targets. 2014;18(6): 651-664

51. Guo X, Namekata K, Kimura A, et al. ASK1 in neurodegeneration. Adv Biol Regul. 2017:

52. Matsuzawa A, Nishitoh $\mathrm{H}$, Tobiume $\mathrm{K}$, et al. Physiological roles of ASK1-mediated signal transduction in oxidative stress- and endoplasmic reticulum stress-induced apoptosis: advanced findings from ASK1 knockout mice. Antioxid Redox Signal. 2002;4(3): 415-425.

53. Harada C, Namekata K, Guo X, et al. ASK1 deficiency attenuates neural cell death in GLAST-deficient mice, a model of normal tension glaucoma. Cell Death Differ. 2010;17(11): 1751-1759.

54. Waak J, Weber SS, Gorner K, et al. Oxidizable residues mediating protein stability and cytoprotective interaction of DJ-1 with apoptosis signal-regulating kinase 1. J Biol Chem. 2009;284(21): 14245-14257.

55. Junn E, Taniguchi H, Jeong BS, et al. Interaction of DJ-1 with Daxx inhibits apoptosis signal-regulating kinase 1 activity and cell death. Proc Natl Acad Sci U S A. 2005;102(27): 9691-9696.

56. Nagakubo D, Taira $T$, Kitaura $H$, et al. DJ-1, a novel oncogene which transforms mouse NIH3T3 cells in cooperation with ras. Biochem Biophys Res Commun. 1997;231(2): 509-513.

57. Hod Y. Differential control of apoptosis by DJ-1 in prostate benign and cancer cells. J Cell Biochem. 2004;92(6): 1221-1233.

58. Liu S, Yang Z, Wei H, et al. Increased DJ-1 and its prognostic significance in hepatocellular carcinoma. Hepatogastroenterology. 2010;57(102-103): 1247-1256.

59. MacKeigan JP, Clements CM, Lich JD, et al. Proteomic profiling drug-induced apoptosis in non-small cell lung carcinoma: identification of RS/DJ-1 and RhoGDIalpha. Cancer Res. 2003;63(20): 6928-6934.

60. Shen Z, Ren Y, Ye D, et al. Significance and relationship between DJ-1 gene and surviving gene expression in laryngeal carcinoma. Eur J Histochem. 2011;55(1): e9.

61. Yuen HF, Chan YP, Law S, et al. DJ-1 could predict worse prognosis in esophageal squamous cell carcinoma. Cancer Epidemiol Biomarkers Prev. 2008;17(12): 3593-3602

62. Bande MF, Santiago M, Blanco MJ, et al. Serum DJ-1/PARK 7 is a potential biomarker of choroidal nevi transformation. Invest Ophthalmol Vis Sci. 2012;53(1): 62-67.

63. Kaliki S, Shields CL, Shields JA. Uveal melanoma: estimating prognosis. Indian J Ophthalmol. 2015;63(2): 93-102.

64. Ramasamy P, Murphy CC, Clynes M, et al. Proteomics in uveal melanoma. Exp Eye Res. 2014;118: 1-12.

65. Pardo M, Garcia A, Thomas B, et al. The characterization of the invasion phenotype of uveal melanoma tumour cells shows the presence of MUC18 and HMG-1 metastasis markers and leads to the identification of DJ-1 as a potential serum biomarker. Int J Cancer. 2006;119(5): 1014-1022.

66. Chen LL, Tian JJ, Su L, et al. DJ-1: a promising marker in metastatic uveal melanoma. J Cancer Res Clin Oncol. 2015;141(2): 315-321.

67. Linge A, Kennedy S, O'Flynn D, et al. Differential expression of fourteen proteins between uveal melanoma from patients who subsequently developed distant metastases versus those who did Not. Invest Ophthalmol Vis Sci. 2012;53(8): 4634-4643. 